O Open Access Full Text Article

\title{
Sappanone A Protects Against Myocardial Ischemia Reperfusion Injury by Modulation of Nrf2
}

This article was published in the following Dove Press journal:

Drug Design, Development and Therapy

\author{
Xiaojing Shi \\ Guizhou Tao \\ Lili Ji \\ Ge Tian
}

Department of Cardiology, The First Affiliated Hospital of Jinzhou Medical University, Jinzhou, People's Republic of China
Correspondence: Xiaojing Shi Department of Cardiology, The First Affiliated Hospital of Jinzhou Medical University, No. 2, the Fifth Section of Renmin Street, Guta District, Jinzhou, Liaoning Province I2100I, People's

Republic of China

Tel/Fax +86-416-4605305

Email shixiaojing3000@I63.com
Background: Oxidative stress is a major contributor to the onset and development of myocardial ischemia reperfusion injury (MIRI). Sappanone A (SA), a homoisoflavanone extracted from the heartwood of Caesalpinia sappan L., has been demonstrated to possess powerful antioxidant activity. Therefore, this study aimed to determine the protective effect of SA on MIRI and investigate its underlying mechanism.

Methods: The rat hearts were isolated and underwent 30-min ischemia, followed by 120min reperfusion to establish the MIRI model, using the Langendorff method. SA was administrated intraperitoneally into rats $1 \mathrm{~h}$ prior to heart isolation. The myocardial infarct size and apoptosis were measured by TTC and terminal deoxynucleotidyl transferase dUTP nick end labeling staining. Myocardial enzyme activity, MDA content and the activities of SOD and GSH-Px were detected by colorimetric spectrophotometric method. Reactive oxygen species (ROS) level was detected by DCFH-DA probe. The change in Keap1/Nrf2 signaling pathway was evaluated by Western blotting.

Results: SA reduced myocardial infarct size and the release of CK-MB and LDH in a dosedependent manner. Moreover, SA improved the recovery of cardiac function, inhibited MIRI-induced apoptosis, repressed the production of ROS and MDA, and enhanced the activities of SOD and GSH-Px. Mechanistically, SA downregulated Keap1, induced Nrf2 nuclear accumulation, and enhanced $\mathrm{Nrf} 2$ transcriptional activity, subsequently resulting in an increase in the expression of the Nrf2 target genes heme oxygenase- 1 and $\mathrm{NAD}(\mathrm{P}) \mathrm{H}$ quinone dehydrogenase 1 . Moreover, SA enhanced the phosphorylation of Nfr2, but the enhancement in Nfr2 phosphorylation was abrogated by PKC or PI3K inhibitor.

Conclusion: Collectively, it was demonstrated that SA prevents MIRI via coordinating the cellular antioxidant defenses and maintaining the redox balance, by modulation of Nrf2 via the PKC or PI3K pathway. Therefore, SA was a potential therapeutic drug for treating MIRI. Keywords: Sappanone A, oxidative stress, apoptosis, myocardial ischemia reperfusion injury, Nrf2

\section{Introduction}

Caesalpinia sappan L., a type of traditional Chinese herb, possesses extensive pharmacological activities, including antioxidant, ${ }^{1,2}$ anti-inflammation $^{3,4}$ and antimicrobial, ${ }^{5}$ Sappanone A (SA), a homoisoflavanone isolated from the dry heartwood of Caesalpinia sappan L., has been reported to have antioxidant and anti-inflammatory activities as well. ${ }^{6}$ Moreover, SA has demonstrated a good effect on the treatment of allergic asthma, ${ }^{7}$ osteoclastogenesis ${ }^{8}$ and melanogenesis ${ }^{9}$, and displays a good application prospect in clinical practice.

Ischemic heart disease is a leading cause of morbidity and mortality globally. ${ }^{10}$ When the myocardium suffers from ischemic insult, especially acute myocardial 
infarction, restoration of blood supply, namely reperfusion therapy is considered as the optimal way to rescue the endangered myocardium. However, reperfusion sometimes itself may abnormally aggravate myocardial damage in clinical practice, a phenomenon known as myocardial ischemia reperfusion injury (MIRI). ${ }^{11}$ It is well accepted that oxidative stress is a major contributor to the onset and development of many pathological states, especially MIRI. ${ }^{12}$ Furthermore, oxidative stress triggered by excessive reactive oxygen species (ROS) is considered as an essential initiator for MIRI. ${ }^{13}$ Therefore, the antioxidant activity of SA suggests its potential use for preventing MIRI.

Nuclear factor E2-associated factor 2 (Nrf2) acts as a key modulator to preserve the redox balance and control the transcriptional expression of downstream antioxidant enzymes. ${ }^{14}$ Keap1-Nrf2 is one of the major signaling pathways to regulate Nrf2 activity. ${ }^{15} \mathrm{Nrf} 2$ activation is demonstrated to decrease myocardial infarct size and propel the recovery of cardiac function following MIRI. ${ }^{16}$ Therefore, it was assumed that SA prevented MIRI, via activating Nrf2 to enhance the antioxidant system.

In the present study, the aim was to investigate the protective effect of SA on MIRI and its modulation of Nrf2 activity. The results indicated that SA pretreatment protected the heart against MIRI in a dose-dependent manner. The cardioprotective effects of SA were involved in the reinforcement of the antioxidant system via the activation of $\mathrm{Nrf} 2$.

\section{Materials and Methods}

\section{Animals and Drugs}

A total of 66 healthy male Wistar rats, weighting $250 \pm 10 \mathrm{~g}$, were obtained from the Department of Laboratory Animal Science of China Medical University (Shenyang, China). All treatment and use of animals in this study adhered to the Guide for the Care and Use of Laboratory Animals (NIH, USA) and was authorized by the Institutional Animal Care and Use Committee of China Medical University.

SA (CAS No. 102067-84-5) (Figure 1), purchased from ChemFaces (Wuhan, China), was dissolved in DMSO (Sigma-Aldrich, St. Louis, MO, USA), which was diluted by saline so that the concentration of DMSO was less than $0.1 \%$ for injection.

\section{Experiment Groups}

The animal experiments were designed as two stages. At the first stage, a total of 48 rats were divided into six

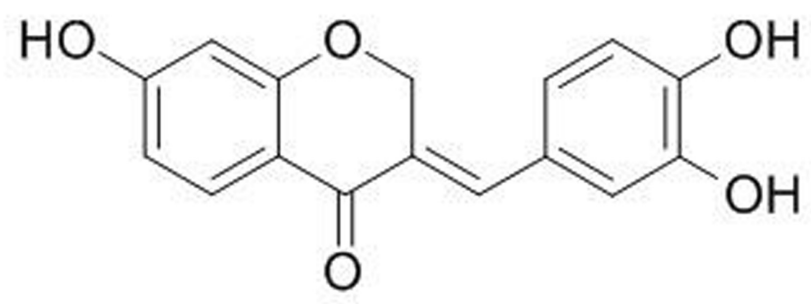

Figure I Chemical structure of Sappanone A.

groups ( $\mathrm{n}=8$ per group), to determine the best concentration of SA treatment as follows. (I) Control group: The isolated heart continuously perfused with Krebs-Henseleit (K-H) solution (in mM: $112 \mathrm{NaCl}, 5 \mathrm{KCl}, 1.2 \mathrm{MgSO}_{4}, 1$ $\mathrm{K}_{2} \mathrm{HPO}_{4}, 1.25 \mathrm{CaCl}_{2}, 25 \mathrm{NaHCO}_{3}, 11$ D-glucose, 0.2 octanoic acid, $\mathrm{pH}=7.4)^{17}$ for $150 \mathrm{~min}$ without ischemia. (II) Ischemia reperfusion (IR) group: The isolated heart underwent 30-min ischemia, followed by 120-min reperfusion. (III) Vehicle group: the rats were intraperitoneally administrated $1 \mathrm{~mL}$ saline (containing 0.1\% DMSO) 1 $\mathrm{h}$ prior to heart isolation. Then, the isolated heart underwent ischemia reperfusion as the IR group. (IV) $10 \mathrm{mg} / \mathrm{kg}$ SA treatment (SA-10) group: $10 \mathrm{mg} / \mathrm{kg}$ SA was intraperitoneally administrated into rats $1 \mathrm{~h}$ prior to heart isolation. Then the isolated heart underwent ischemia reperfusion, as with the IR group. (V) $20 \mathrm{mg} / \mathrm{kg}$ SA treatment (SA-20) group: Rats were treated with $20 \mathrm{mg} / \mathrm{kg} \mathrm{SA}$ and underwent ischemia reperfusion, as described for the SA-10 group. (VI) $40 \mathrm{mg} / \mathrm{kg}$ SA treatment (SA-40) group: Rats were treated with $40 \mathrm{mg} / \mathrm{kg} \mathrm{SA}$ and underwent ischemia reperfusion, as described in the SA-10 group.

In the second stage, a total of 54 rats were divided into six groups ( $\mathrm{n}=6$ per group) to explore the underlying mechanism: (i) Control group: the same as the first stage. (ii) IR group: the same as the first stage. (iii) The best concentration of SA treatment group: Rats treated with the best concentration of SA, determined in the first stage, and underwent ischemia reperfusion at the same as the first stage. (iv) SA + chelerythrine (CHE) group: CHE, a PKC inhibitor, was intraperitoneally administrated into rats at the dose of $1 \mathrm{mg} / \mathrm{kg} 15 \mathrm{~min}$ prior to SA administration, based on a previous study. ${ }^{18}$ (v) SA + (LY294002) LY group: LY294002, a PI3K inhibitor was intraperitoneally administrated into rats at the dose of $0.3 \mathrm{mg} / \mathrm{kg} 15 \mathrm{~min}$ prior to SA administration, based on a previous study. ${ }^{19}$ (vi) SA + ML385 group: ML385, a Nrf2 inhibitor, was intraperitoneally injected at the dose of $30 \mathrm{mg} / \mathrm{kg}, 30$ mins prior to SA administration, based on a previous study. ${ }^{20}$ 


\section{MIRI Created in an Isolated Rat Heart Model}

Rats were anesthetized by intravenous injection of pentobarbital sodium (100 mg/kg). Heparin (1500 IU/kg) was simultaneously given by an intravenous injection to prevent intracoronary thrombus formation. The rat heart was rapidly removed from the thoracic cavity and subsequently placed into a $4^{\circ} \mathrm{C}$ heparinized Krebs-Henseleit (K-H) solution. Afterwards, the isolated heart was hung on a Langendorff perfusion device from the root of the aorta and perfused with $95 \% \mathrm{O}_{2}+5 \% \mathrm{CO}_{2}$-saturated $\mathrm{K}-\mathrm{H}$ solution, under a constant pressure of $75 \mathrm{mmHg}$ at $37^{\circ} \mathrm{C}$. The fluid-filled latex balloon was inserted in the left ventricle via the left atrium, and was connected to a pressure transducer. The heart rate, left ventricular-developed pressure (LVDP), positive first-order derivative of ventricular pressure $(+\mathrm{dp} / \mathrm{dt})$, and negative first-order derivative of ventricular pressure (-dp/dt) were recorded and analyzed via a homodynamic system (MP150; BIOPAC Systems, Inc., Goleta, CA, USA). All hearts underwent $30 \mathrm{~min}$ of ischemia, followed by $120 \mathrm{~min}$ of reperfusion.

\section{Measurement of Infarct Size}

The hearts were collected at the end of reperfusion, and frozen at $-20^{\circ} \mathrm{C}$ for $1 \mathrm{~h}$. The frozen hearts were cut into $1-\mathrm{mm}$ sections and incubated in $1 \%$ triphenyltetrazolium chloride (TTC) solution at $37^{\circ} \mathrm{C}$ for $10 \mathrm{~min}$. The sections were subsequently fixed by $4 \%$ paraformaldehyde for 24 $\mathrm{h}$ and photographed using a digital camera.

\section{Myocardial Enzyme Test}

When myocardial damage occurs, lactate dehydrogenase (LDH) and creatine kinase-MB (CK-MB) will release into the coronary effluent. The coronary effluent was collected at the end of reperfusion and the activities of LDH and CK-MB were detected using the LDH Assay kit and CKMB Assay kit (Jiancheng Biotech Co., Ltd, Nanjing, China), following to the manufacturer's instructions.

\section{Terminal Deoxynucleotidyl Transferase dUTP Nick End Labeling (TUNEL) Assay}

To detect apoptosis in the myocardium, the TUNEL assay was performed using the In Situ Cell Death Detection kit (Roche, USA), following the manufacturer's protocols. Each section was observed under a light microscope and images were captured. Apoptotic cells were calculated in five random high-magnified fields per section by two independent observers.

\section{Measurement of ROS Content}

The hearts were harvested at the end of reperfusion and washed in ice-cold PBS solution. Single-cell suspension of the myocardium was prepared as previously described. ${ }^{21}$ Single-cell suspension was incubated with 2,7-dichlorofluorescein diacetate (DCFH-DA), a sensitive ROS probe (Jiancheng Biotech Co., Ltd, Nanjing, China), at $37^{\circ}$ for $1 \mathrm{~h}$, and then centrifuged at $1000 \mathrm{~g}$ for $10 \mathrm{~min}$ to collect DCFH-DA-stained cardiomyocytes. DCFH-DA-stained cardiomyocytes were resuspended and fluorescence intensity was detected using the Automatic Fluorescence Microplate Reader, according to the manufacturer's instructions.

\section{Measurement of Malondialdehyde (MDA), Superoxide Dismutase (SOD) and (Glutathione Peroxidase) GSH-Px}

The hearts were harvested at the end of reperfusion and washed in ice-cold PBS solution. The heart tissues were cut into small pieces, homogenized with RIPA lysis buffer, centrifuged at $12,000 \mathrm{~g}$ for $10 \mathrm{~min}$, and the supernatant was collected. The MDA content and the activities of SOD and GSH-Px were detected using the MDA Detection kit (Jiancheng Biotech Co., Ltd, Nanjing, China), SOD Detection kit (Jiancheng Biotech Co., Ltd, Nanjing, China) and GSH-PX Detection kit (Jiancheng Biotech Co., Ltd, Nanjing, China), according to the manufacturer's instructions.

\section{Measurement Nrf2 Transcription Activity} To measure the transcription activity of Nrf2, the nuclear extract lysates were obtained from the harvested myocardium, using the Nuclear Extraction kit (cat. no. ab113474; Abcam, Cambridge, UK), according to the manufacturer's protocols. The transcription activity of Nrf2 in nuclear extract lysates was detected by enzyme-linked immunosorbent assay, using the Nrf2 Transcription Factor Assay kit (cat. no. ab207223; Abcam), according to the manufacturer's instructions.

\section{Western Blotting Analysis}

Proteins were extracted from cell lysates, using RIPA lysis buffer, and the protein concentration was measured using the Enhanced BCA Protein Assay kit (Beyotime Institute of Biotechnology), following the manufacturer's recommendations. Proteins were denatured by heat, and then separated by SDS-PAGE electrophoresis, and finally transferred to PVDF membranes. The membranes were blocked using $1 \%$ bovine serum albumin solution for $1 \mathrm{~h}$ at room temperature, and then incubated with primary antibodies, 
including anti-cleaved caspase-3 (1:1000; Abcam), antiKeap1 (1:1000; Abcam), anti-Nrf2 (1:1000; Abcam), anti-p-Nrf2 (1:1000; Abcam), anti-heme oxygenase-1 $(1: 1000 ;$ Abcam), anti-NAD $(\mathrm{P}) \mathrm{H}$ quinone dehydrogenase 1 (1:1000; Abcam) and anti- $\beta$-actin $(1: 1000 ;$ Zhongshan Jinqiao Biotechnology, Beijing, China) at $4{ }^{\circ} \mathrm{C}$ overnight, followed by incubation with HRP-conjugated secondary antibody (1:5000; Zhongshan Jinqiao Biotechnology, Beijing, China) at room temperature for $30 \mathrm{~min}$. Protein bands were detected using a Immun-Star HRP kit (BioRad), following the manufacturer's protocols. Relative densitometry was analyzed using the Image $\mathrm{J} 2 \mathrm{x}$ analysis software (National Institutes of Health, USA).

\section{Statistical Analysis}

Data are expressed as the mean \pm standard deviation (SD). To analyze the differences between two groups, Student's $t$-test was conducted, and the differences between multiple groups were analyzed using one-way analysis of variance. $\mathrm{P}<0.05$ indicated a statistically significant difference. All statistical analysis was carried out using SPSS version 17.0 software (SPSS Inc., Chicago, IL).

\section{Results}

\section{SA Mitigates MIRI in a Dose-Dependent \\ Manner}

Firstly, the effect of the different doses of SA (10, 20 and $40 \mathrm{mg} / \mathrm{kg}$ ) pretreatment on the myocardial infarct size and the release of myocardial enzymes were examined. The results of TTC staining showed that the myocardial infarct size was significantly decreased in all SA treatment groups compared with the IR group. Moreover, the myocardial infarct size in the SA-20 group was less compared with the SA-10 treatment group but indicated no significant difference
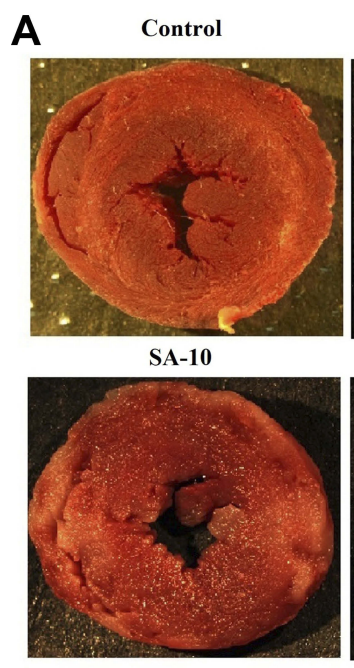

$\mathbf{B}$

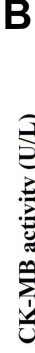

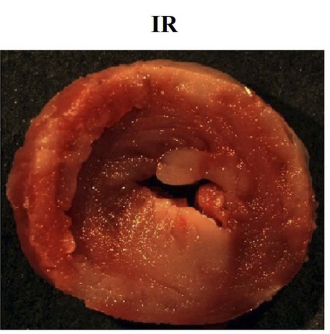

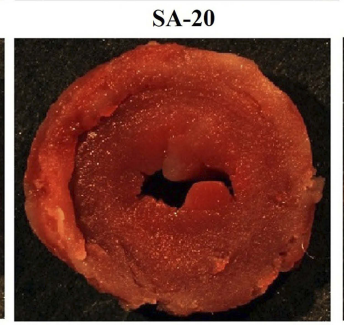

$* *$

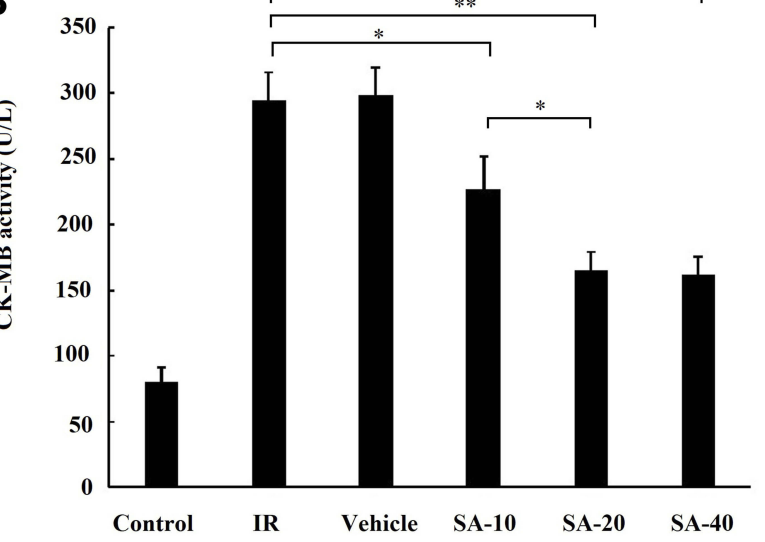

Vehicle
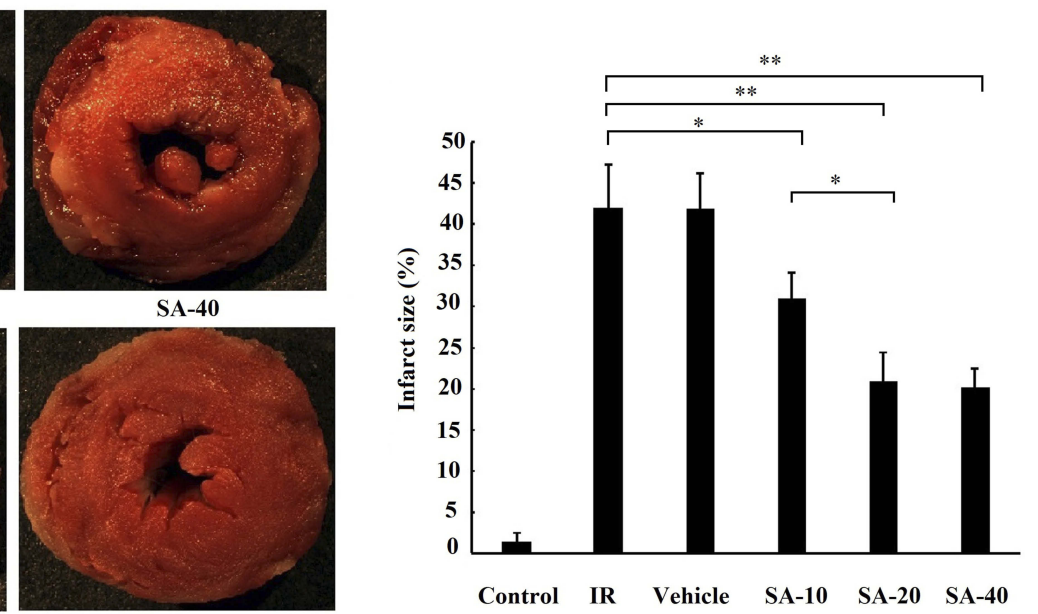

C

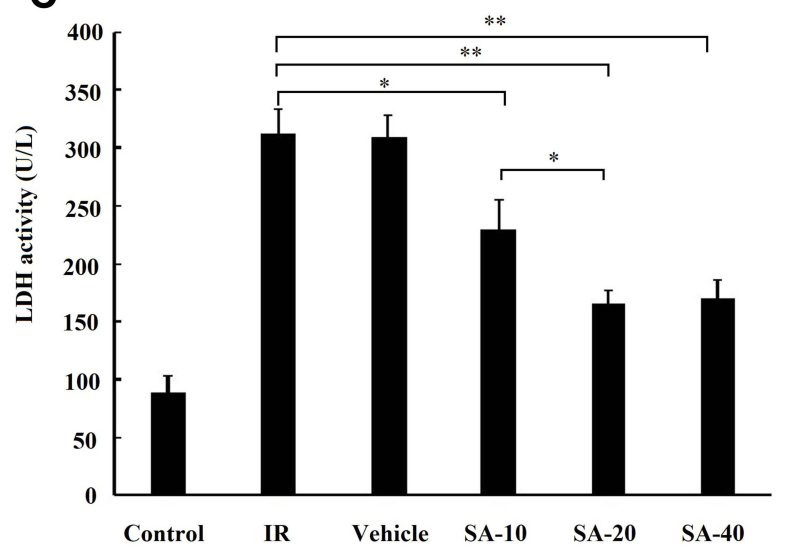

Figure 2 Sappanone A (SA) mitigated myocardial ischemia reperfusion injury in a dose-dependent manner. SA at the concentration of 10 , 20 and 40 mg/kg was intraperitoneally administrated into rats I $\mathrm{h}$ prior to heart isolation, and then the hearts were isolated that underwent 30-min ischemia, followed by 120 -min reperfusion. (A) Myocardial infarct size was measured by TTC staining. (B) The creatine kinase-MB (CK-MB) and (C) lactate dehydrogenase (LDH) activity in the coronary effluent were measured by spectrophotometry. Data are presented as the mean \pm standard deviation, $\mathrm{n}=8$. $* \mathrm{P}<0.05, * * \mathrm{P}<0.0 \mathrm{I}$. 
(Figure 2A). The results of CK-MB and LDH also displayed similar results to those observed with the myocardial infarct size (Figure $2 \mathrm{~B}$ and $\mathrm{C}$ ). Taken together, these results suggested that SA mitigated MIRI in a dose-dependent manner and $20 \mathrm{mg} / \mathrm{kg} \mathrm{SA}$ was chosen for further investigation, due to optimal cardio-protective manifestation.

\section{SA Ameliorated Cardiac Function After Ischemia Reperfusion}

The influence of SA treatment on the recovery of cardiac function following ischemia reperfusion was further investigated. The result of cardiac function showed that SA treatment remarkably improved the values of LVDP and $\pm \mathrm{dp} / \mathrm{dt}$ at 30,60 and $120 \mathrm{~min}$ of reperfusion compared with the IR group (Figure 3B-D). However, there is no significant difference in the value of the heart rate between SA group and IR group at all time-points (Figure 3A). These results suggested that SA improved the recovery of cardiac function, following ischemia reperfusion.

\section{SA Inhibits Ischemia Reperfusion-Induced Myocardial Apoptosis}

To investigate the effect of SA treatment on ischemia reperfusion-induced myocardial apoptosis, TUNEL staining and Western blotting were used to detect the number of apoptotic cells and the expression of cleaved caspase-3, respectively. The results indicated that the apoptosis rate was significantly decreased in the SA treatment group compared with the IR group (Figure 4A). Furthermore, the expression of cleaved caspase -3 was suppressed by
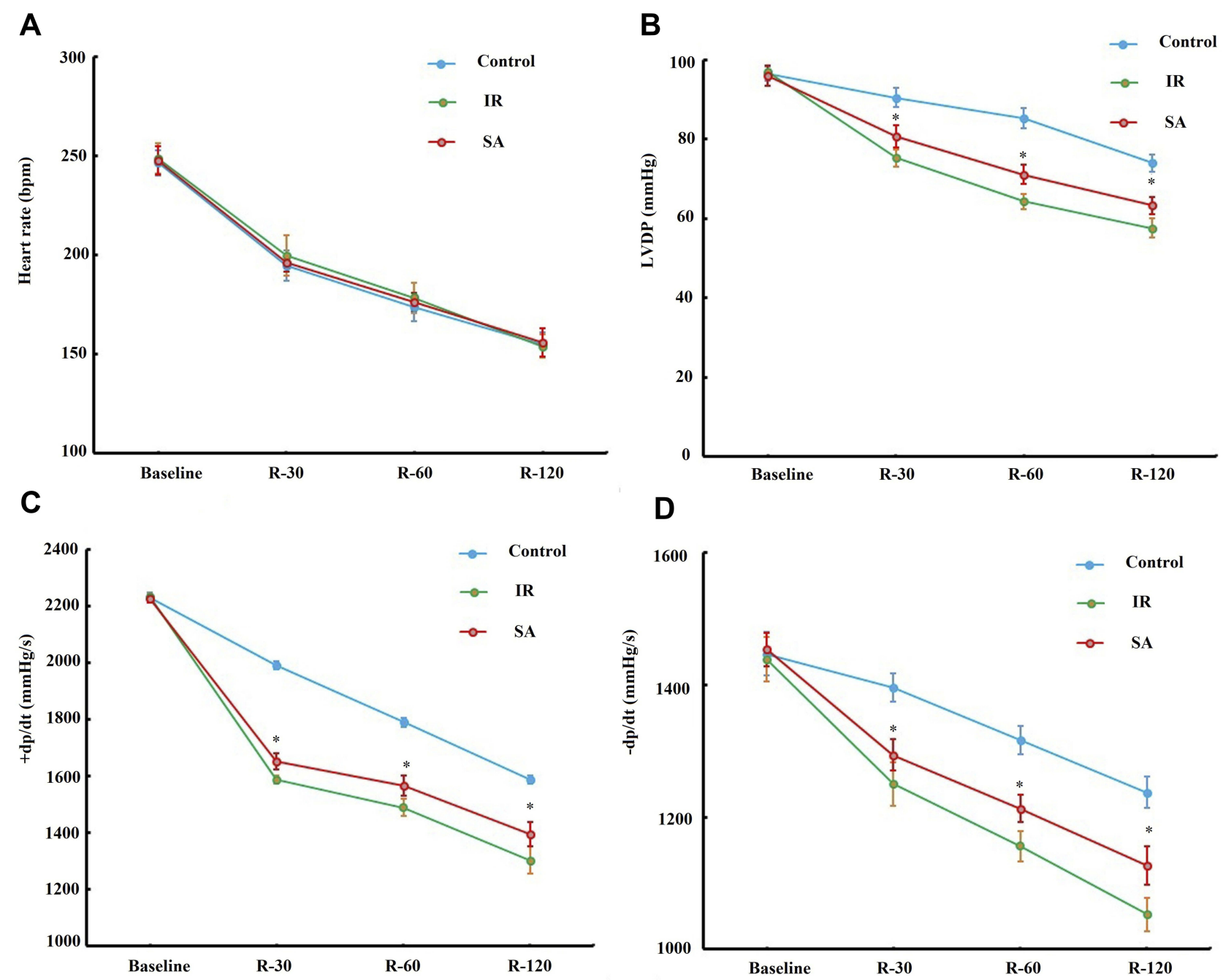

Figure 3 Sappanone A (SA) ameliorated cardiac function following ischemia reperfusion (IR). SA ( $20 \mathrm{mg} / \mathrm{kg}$ ) was intraperitoneally administrated into rats I h prior to heart isolation, and then the hearts were isolated and underwent 30-min ischemia, followed by 120-min reperfusion. The changes in heart rate (A), left ventricular-developed pressure (LVDP) (B), positive first-order derivative of ventricular pressure $(+\mathrm{dp} / \mathrm{dt})(\mathbf{C})$, and negative first-order derivative of ventricular pressure $(-\mathrm{dp} / \mathrm{dt})(\mathbf{D})$ were recorded I min before ischemia (baseline), after 30 (R-30), 60 (R-60) and I 20 (R-120) min of reperfusion. Data are presented as the mean \pm standard deviation, $n=6$. *P<0.05. 
SA treatment (Figure 4B). Taken together, these results suggested that $\mathrm{SA}$ inhibited ischemia reperfusion-induced myocardial apoptosis.

\section{SA Alleviates Ischemia Reperfusion-Induced Oxidative Stress Injury}

To investigate the effect of SA treatment on ischemia reperfusion-induced oxidative stress injury, the changes of ROS, MDA, SOD and GSH-Px were measured. As shown in Figure 5A and B, SA treatment significantly decreased the content of ROS and MDA in ischemic myocardium. Meanwhile, SA enhanced the activities of SOD and GSH$\mathrm{Px}$ in ischemic myocardium (Figure $5 \mathrm{C}$ and $\mathrm{D}$ ). Taken together, these results suggested that SA alleviated ischemia reperfusion-induced oxidative stress injury.

\section{SA Activated Keapl-Nrf2 Signal Pathway}

To further determine whether SA treatment activated Keap1-Nrf2 signal pathway, the changes in the expression of Keap1 and Nrf2 by SA treatment were evaluated. The result of Western blotting showed that SA treatment downregulated the expression of Keap1 (Figure 6A), decreased the level of Nrf2 in the cytoplasm (Figure 6B), and increased the level of Nrf2 in the nucleus (Figure 6C). Also, the result from the ELISA assay indicated the activation of transcription activity by SA treatment (Figure 6D). Meanwhile, SA treatment upregulated the Nrf2 target genes (HO-1 and NQO1) expressions (Figure 6E and F). Taken together, these results suggested that SA activated the Keap1-Nrf2 signal pathway in the MIRI condition.

\section{SA Regulated the Phosphorylation of $\mathrm{Nfr} 2$}

To further explore how SA regulates the nuclear translocation of Nfr2, the change in the phosphorylation of Nfr2 was evaluated using Western blotting. As shown in Figure 7, the phosphorylation level of $\mathrm{Nfr} 2$ was remarkably enhanced by SA. However, the enhancement in Nfr2 phosphorylation was abrogated by PKC or PI3K inhibitor. Therefore, these results suggested that $\mathrm{SA}$ enhanced the phosphorylation of Nfr2 via PKC or PI3K pathway.

\section{The Cardioprotective Effects of SA Was Abrogated by Inhibition of Nfr2}

Whether the cardioprotective effects of SA were rescued by the inhibition of Nfr2, through ML385 treatment, was further

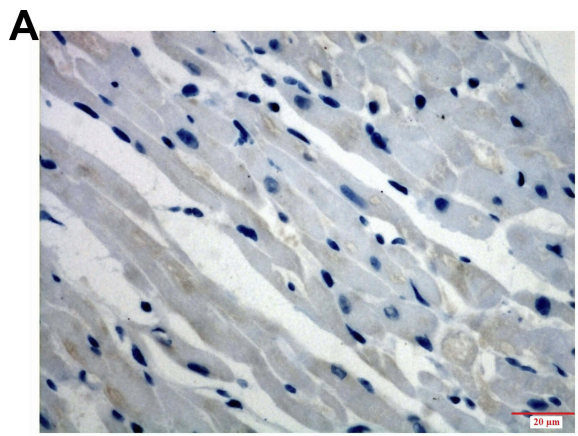

Control



SA

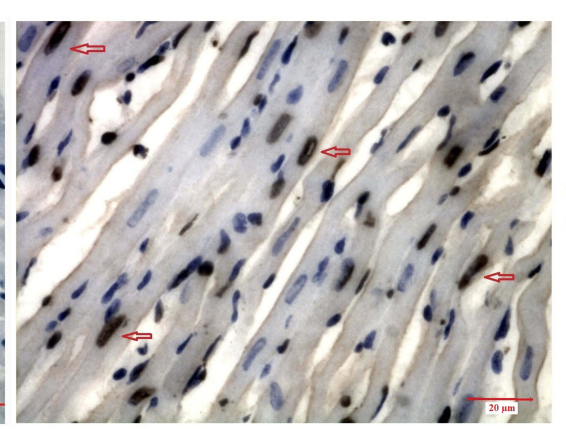

IR

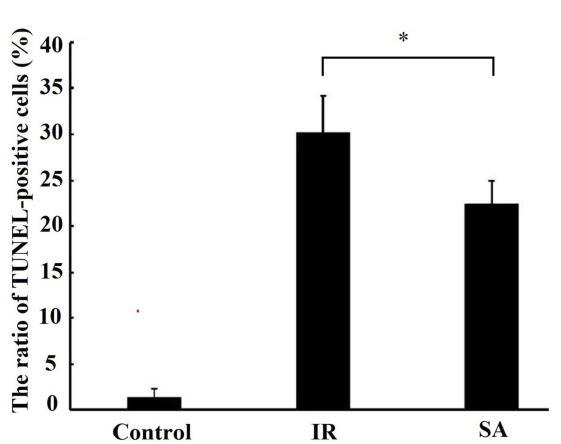

B
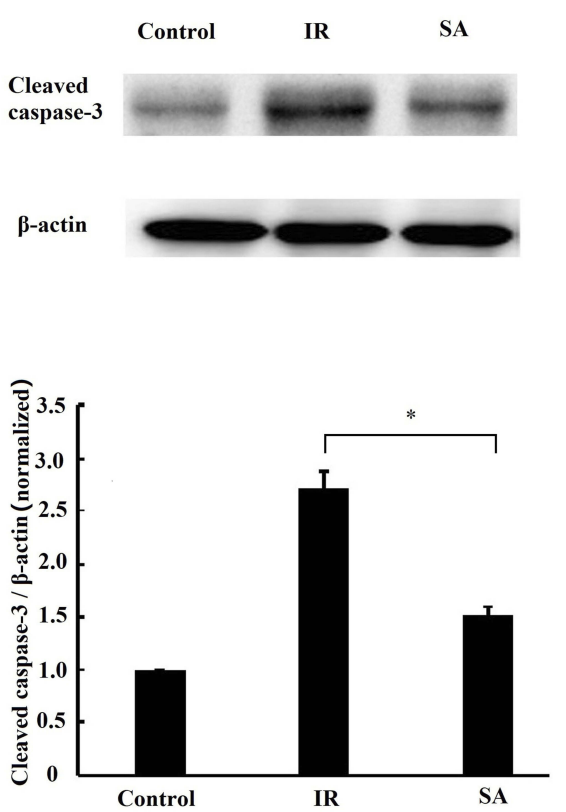

Figure 4 Sappanone A (SA) inhibited ischemia reperfusion (IR)-induced myocardial apoptosis. SA (20 mg/kg) was intraperitoneally administrated into rats I h prior to heart isolation, and then the hearts were isolated that underwent 30 -min ischemia, followed by 120 -min reperfusion. (A) Myocardial apoptosis was measured by terminal deoxynucleotidyl transferase dUTP nick end labeling staining $(x 400)(n=6$ per group). The apoptotic cell nuclei were stained brown and the living cell nuclei were stained blue. Scale bar $=20 \mu \mathrm{m}$. (B) The expression of cleaved caspase-3 was measured by Western blotting $(\mathrm{n}=3)$. Data are expressed as the mean \pm standard deviation (SD). $* \mathrm{P}<0.05$. 


\section{A}

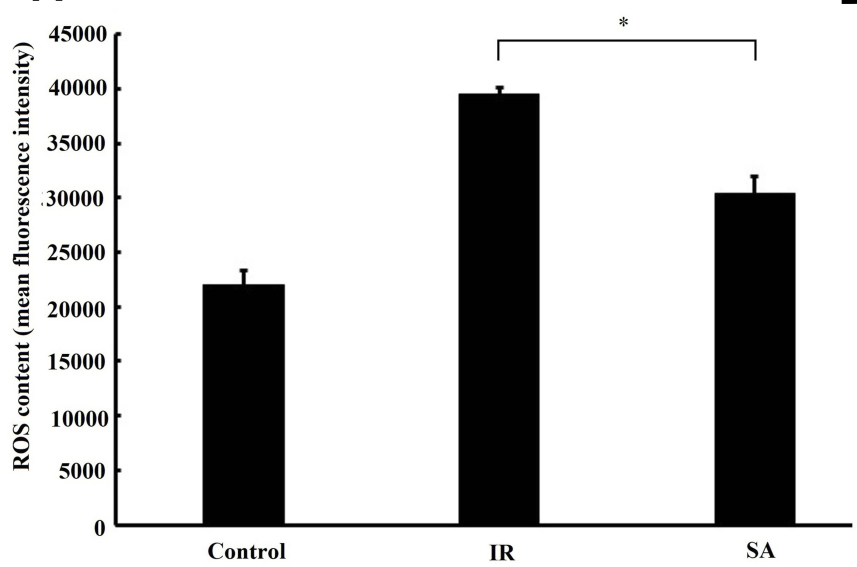

C

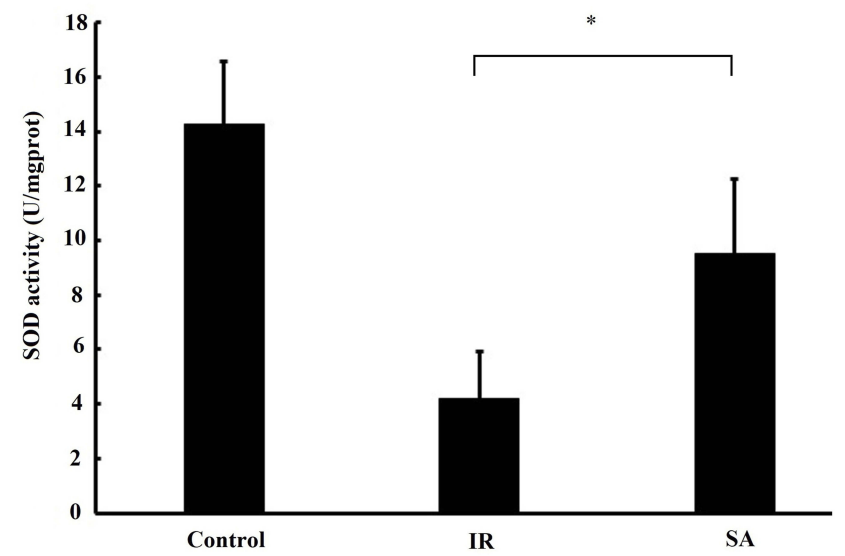

B

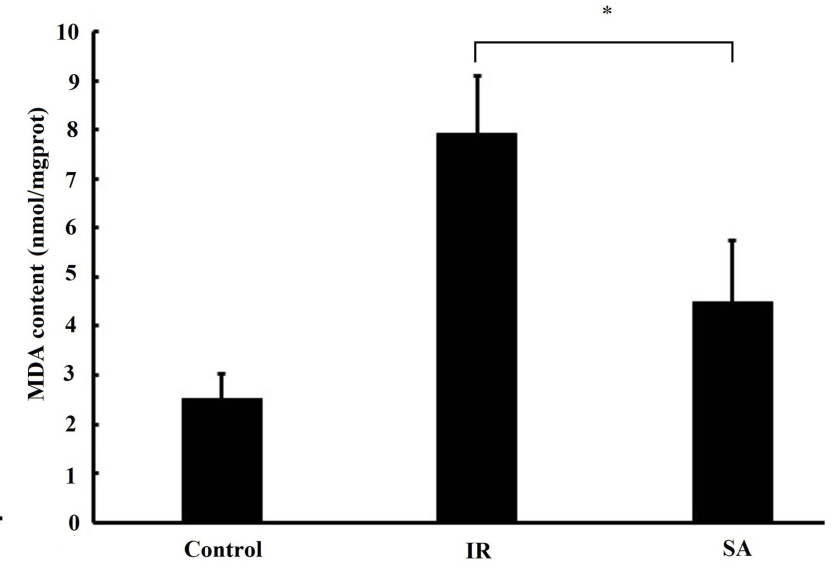

D

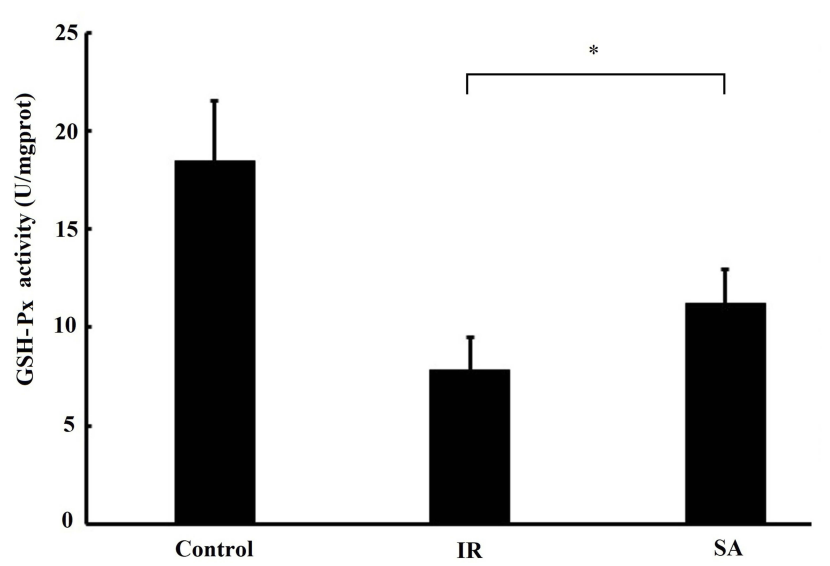

Figure 5 Sappanone A (SA) alleviated ischemia reperfusion (IR)-induced oxidative stress injury. SA (20 mg/kg) was intraperitoneally administrated into rats I h prior to heart isolation, and then the hearts were isolated and underwent 30-min ischemia, followed by I20-min reperfusion. (A) Reactive oxygen species (ROS) level was detected by DCFH-DA probe. Mean fluorescence intensity reflected the ROS level. The content of malondialdehyde (MDA) (B) and activities of superoxide dismutase (SOD) (C) and glutathione peroxidase $(\mathrm{GSH}-\mathrm{Px})(\mathrm{D})$ were detected by colorimetric spectrophotometric method. Data are expressed as the mean \pm standard deviation, $\mathrm{n}=6$. $* \mathrm{P}<0.05$.

tested. The results showed that the reduction of infarct size (Figure 8A) and the decrease of CK-MB and LDH (Figure 8B and C) by SA were reversed by the Nfr2 inhibitor (ML385), which suggested that the cardioprotective effects of SA were abrogated by the inhibition of $\mathrm{Nfr} 2$ and indicates that SA prevents against MIRI via Nfr2 conversely.

\section{Discussions}

MIRI not only aggravates myocardial necrosis and apoptosis but also results in the deterioration of heart function, even manifesting myocardial stunning. ${ }^{13}$ In the present study, it was found that SA pretreatment led to the reduction of myocardial necrosis and apoptosis, and improved the recovery of cardiac function following MIRI. To our knowledge, this is the first study to demonstrate the cardioprotective effects of SA on MIRI. The dried heartwood of Caesalpinia sappan L. has been used to accelerate blood circulation and remove blood stasis in China for a long time. ${ }^{22}$ Notably, the ethanolic extract of Caesalpinia sappan $L$. heartwood has been reported to protect the brain against ischemia reperfusion injury in a middle cerebral artery occlusion (MCAO) rat model. ${ }^{23}$ However, it is not clear exactly which ingredient plays an anti-ischemia role. These results suggested that SA is a major ingredient of Caesalpinia sappan $L$. that accounts for the anti-ischemia effect.

Oxidative stress occurs when the balance between the generation of ROS and the antioxidant defense systems is disturbed. ${ }^{24}$ Prolonged ischemia following reperfusion will generate a burst of ROS that includes hydrogen peroxide (H2O2), the superoxide radical anion, the hydroxyl radical $(\mathrm{OH} \bullet)$ and peroxynitrite anion (ONOO-). These increased ROS will further mediate membrane lipid peroxidation reaction and generate MDA. Meanwhile, the antioxidant enzyme system, such as the activities of SOD and GSHPx, is also severely weakened. ${ }^{25}$ Therefore, anti-oxidative 

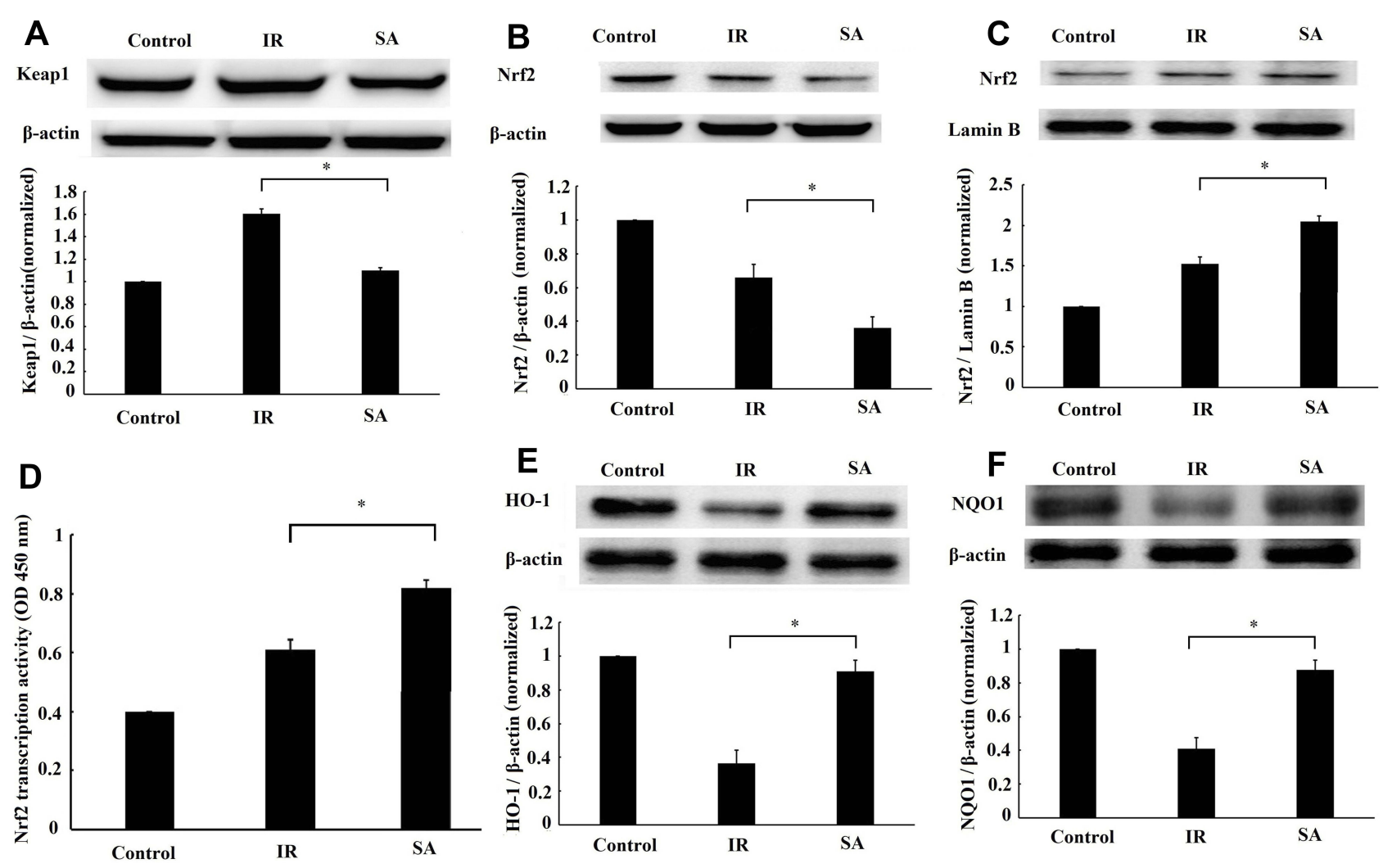

Figure 6 Sappanone A (SA) activated Keapl-Nrf2 signal pathway in ischemia reperfusion (IR)-myocardium. SA (20 mg/kg) was intraperitoneally administrated into rats I $\mathrm{h}$ prior to heart isolation, and then the hearts were isolated and underwent 30-min ischemia, followed by 120-min reperfusion. The expression of Keapl(A), Nrf2 in the cytoplasm (B) or nuclei (C) were detected by Western blotting. (D) The transcriptional activity of Nrf2 was measured by enzyme-linked immunosorbent assay. The expression of Nrf2 target genes heme oxygenase-I (E) and $\mathrm{NAD}(\mathrm{P}) \mathrm{H}$ quinone dehydrogenase I (F) detected by Western blotting. Data are expressed as the mean \pm standard deviation (SD), $n=3$. ${ }^{*} \mathrm{P}<0.05$.

stress is considered as an important target for MIRI treatment and mounting evidence supported the use of antioxidants to prevent MIRI. ${ }^{26}$ In the present study, it was found that SA pretreatment reduced the ROS and MDA content, and enhanced the activities of SOD and GSH-Px in ischemic myocardium. Kang et al also reported that SA inhibited MDA production and upregulated the activities of SOD and GSH-Px in cisplatin-induced kidney injury. ${ }^{6}$ This evidence indicated that SA could coordinate the cellular antioxidant defenses and maintain the redox balance during MIRI.

Nrf2 physiologically localizes in the cytoplasm via binding to its cytosolic physiological inhibitor, Keap1, and the latter facilitates Nrf2 ubiquitination and subsequent proteasomal degradation by the Cu3-Rbk1 complex. ${ }^{27}$ When oxidative stress occurs, Keap1 conformation will alter, which leads to the dissociation of Nrf2 and Keap1, followed by the entry of Nrf2 into the nucleus without ubiquitination and combines with the ARE to trigger the transcription of endogenous protective genes, ${ }^{28}$ such as HO-1 and NQO1 ${ }^{29}$ In this study, it was found that SA downregulated Keap1, induced Nrf2 nuclear accumulation and enhanced its transcriptional activity, subsequently resulting in an increase in the expression of the Nrf2 target genes HO-1 and NQO1 in the ischemic myocardium. Similarly, SA was also reported to induce the nuclear translocation of $\mathrm{Nrf} 2$ and HO-1 expression in lipopolysaccharide-treated RAW264.7 cells by Lee et $\mathrm{al}^{30}$ Taken together, this evidence suggested that SA alleviated MIRI, partly via the activation of Nrf2.

Although mounting evidence has demonstrated that SA could induce the nuclear translocation of Nrf2,, 30 its underlying mechanism is not well understood. It is well accepted that the phosphorylation of $\mathrm{Nrf} 2$ by protein kinases, such as $\mathrm{PKC}$ and PI3K, promotes the nuclear translocation of Nrf2 by dissociating from Keap $1 .{ }^{14}$ In the present study, it was also demonstrated that SA enhanced the phosphorylation of $\mathrm{Nfr} 2$, via the PKC or PI3K pathway. These findings may give a new insight into how SA induces the nuclear translocation of Nrf2. However, it must be acknowledged that a limitation exists in this study. Although the isolated rat heart model generated by the Langendorff methods is stabilized and reproducible, this ex vivo model could not 
A

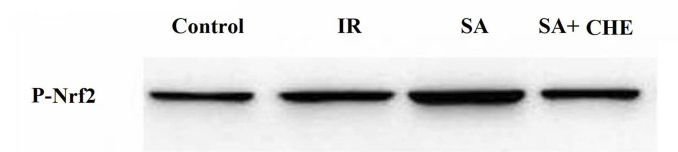

$\beta$-actin
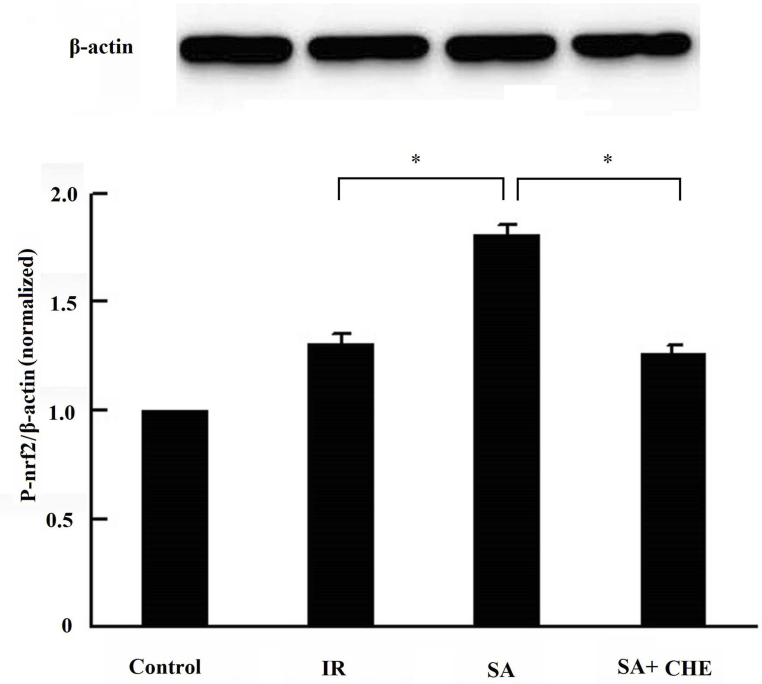

B
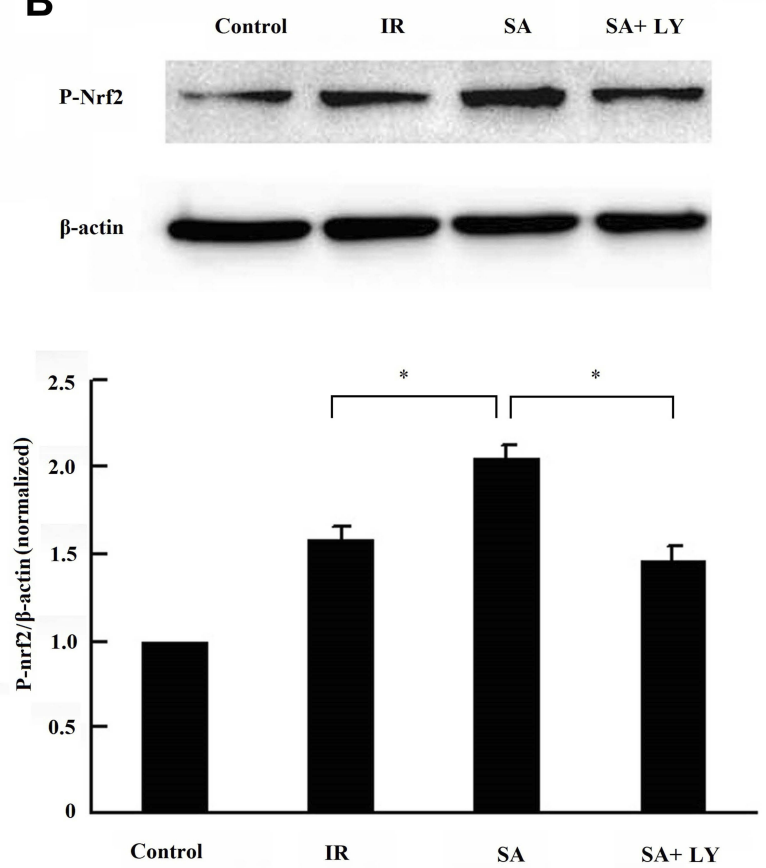

Figure 7 Sappanone A (SA) regulated the phosphorylation of Nfr2. SA (20 mg/kg) was intraperitoneally administrated into rats I h prior to heart isolation, and then the rat hearts were isolated and underwent 30-min ischemia, followed by 120 -min reperfusion. (A) Chelerythrine (CHE), a PKC inhibitor, was intraperitoneally administrated into rats at the dose of $\mathrm{I} \mathrm{mg/kg,} 15$ min prior to SA administration. (B) LY294002 (LY), a PI3K inhibitor was intraperitoneally administrated into rats at the dose of $0.3 \mathrm{mg} / \mathrm{kg}, 15 \mathrm{~min}$ prior to SA administration. The phosphorylation of Nfr2 (P-Nfr2) was detected by Western blotting. Data are expressed as the mean \pm standard deviation, $\mathrm{n}=3$. $* \mathrm{P}<0.05$.

A



B

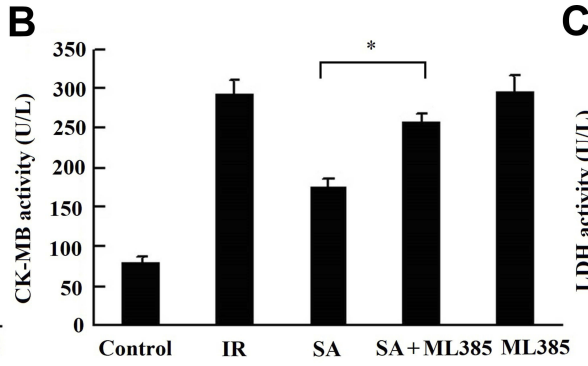

C

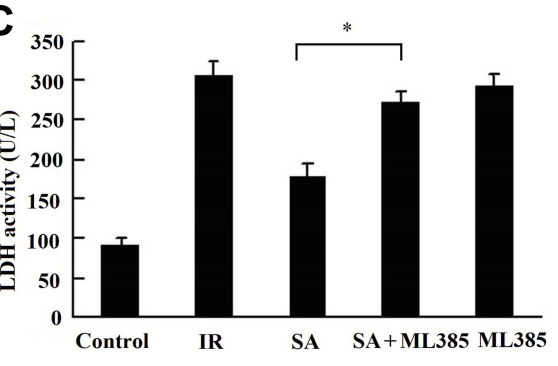

Figure 8 The cardioprotective effects of SA were abrogated by the inhibition of Nfr2. SA (20 mg/kg) was intraperitoneally administrated into rats I h prior to heart isolation, and then the hearts were isolated and underwent 30-min ischemia, followed by 120 -min reperfusion. ML385, a Nrf2 inhibitor, was intraperitoneally injected at the dose of $30 \mathrm{mg} / \mathrm{kg} 30 \mathrm{~min}$, prior to SA administration. (A) Myocardial infarct size was measured by TTC staining. (B) The creatine kinase-MB (CK-MB) and (C) lactate dehydrogenase $(\mathrm{LDH})$ activities in the coronary effluent were measured by spectrophotometry. Data are presented as the mean \pm standard deviation, $\mathrm{n}=6$. $* \mathrm{P}<0.05$.

perfectly mimic the pathophysiological process of MIRI, due to the lack of neural and humoral regulation. ${ }^{17}$ Therefore, the protective effect of SA on MIRI should be further confirmed in vivo.

Collectively, the present study demonstrated that SA prevents MIRI by coordinating the cellular antioxidant defenses and maintaining the redox balance, by the modulation of Nrf2. Thus, SA is a potential therapeutic drug for treating MIRI.

\section{Acknowledgements}

This study was funded by National Key R\&D Program of China (grant no. 2016YFC1301303).

\section{Author Contributions}

All authors contributed to data analysis, drafting or revising the article, gave final approval of the version to be published, and agree to be accountable for all aspects of the work. 


\section{Disclosure}

The authors report no conflicts of interest in this work.

\section{References}

1. Safitri R, Tarigan P, Freisleben HJ, Rumampuk RJ, Murakami A. Antioxidant activity in vitro of two aromatic compounds from Caesalpinia sappan L. Biofactors. 2003;19(1-2):71-77. doi:10.1002/ biof.v19:1/2

2. Choi BM, Lee JA, Gao SS, et al. Brazilin and the extract from Caesalpinia sappan L. protect oxidative injury through the expression of heme oxygenase-1. Biofactors. 2007;30(3):149-157. doi:10.1002/ biof. 5520300302

3. Tewtrakul S, Tungcharoen P, Sudsai T, Karalai C, Ponglimanont C, Yodsaoue O. Antiinflammatory and wound healing effects of Caesalpinia sappan L. Phytother Res. 2015;29(6):850-856. doi:10.1002/ ptr.v29.6

4. Jung EG, Han KI, Kwon HJ, et al. Anti-inflammatory activity of sappanchalcone isolated from Caesalpinia sappan L. in a collagen-induced arthritis mouse model. Arch Pharm Res. 2015;38 (6):973-983. doi:10.1007/s12272-015-0557-z

5. Nirmal NP, Panichayupakaranant P. Antioxidant, antibacterial, and anti-inflammatory activities of standardized brazilin-rich Caesalpinia sappan extract. Pharm Biol. 2015;53(9):1339-1343. doi:10.3109/ 13880209.2014.982295

6. Kang L, Zhao H, Chen C, Zhang X, Xu M, Duan H. Sappanone A protects mice against cisplatin-induced kidney injury. Int Immunopharmacol. 2016;38:246-251. doi:10.1016/j.intimp.2016.05.019

7. Liu X, Yu D, Wang T. Sappanone A attenuates allergic airway inflammation in ovalbumin-induced asthma. Int Arch Allergy Immunol. 2016;170(3):180-186. doi:10.1159/000448331

8. Choo YY, Tran PT, Min BS, et al. Sappanone A inhibits RANKL-induced osteoclastogenesis in BMMs and prevents inflammation-mediated bone loss. Int Immunopharmacol. 2017;52:230-237. doi:10.1016/j.intimp. 2017.09.018

9. Chang TS, Chao SY, Ding HY. Melanogenesis inhibition by homoisoflavavone sappanone A from Caesalpinia sappan. Int $J$ Mol Sci. 2012;13(8):10359-10367. doi:10.3390/ijms130810359

10. Benjamin EJ, Virani SS, Callaway CW, et al. Heart disease and stroke statistics-2018 update: a report from the American Heart Association. Circulation. 2018;137(12):67-492.

11. Davidson SM, Ferdinandy P, Andreadou I, et al. Multitarget strategies to reduce myocardial ischemia/reperfusion injury: JACC review topic of the week. J Am Coll Cardiol. 2019;73(1):89-99. doi:10.1016/j.jacc.2018.09.086

12. Hausenloy DJ, Yellon DM. Myocardial ischemia-reperfusion injury: a neglected therapeutic target. J Clin Invest. 2013;123(1):92-100. doi:10.1172/JCI62874

13. Yellon DM, Hausenloy DJ. Myocardial reperfusion injury. $N$ Engl $J$ Med. 2007;357(11):1121-1135. doi:10.1056/NEJMra071667

14. Shen Y, Liu X, Shi J, Wu X. Involvement of Nrf2 in myocardial ischemia and reperfusion injury. Int $J$ Biol Macromol. 2019;125:496-502. doi:10.1016/j.ijbiomac.2018.11.190

15. Tu W, Wang H, Li S, Liu Q, Sha H. The anti-inflammatory and anti-oxidant mechanisms of the keap1/Nrf2/ARE signaling pathway in chronic diseases. Aging Dis. 2019;10(3):637-651. doi:10.14336/ AD.2018.0513
16. Shanmugam G, Narasimhan M, Tamowski S, Darley-Usmar V, Rajasekaran NS. Constitutive activation of Nrf2 induces a stable reductive state in the mouse myocardium. Redox Biol. 2017;12:937-945. doi:10.1016/j.redox.2017.04.038

17. Herr DJ, Aune SE, Menick DR. Induction and assessment of ischemia-reperfusion injury in Langendorff-perfused rat hearts. $J$ Vis Exp. 2015;101:e52908.

18. Lai LN, Zhang XJ, Zhang XY, et al. Lazaroid U83836E protects the heart against ischemia reperfusion injury via inhibition of oxidative stress and activation of PKC. Mol Med Rep. 2016;13:3993-4000. doi:10.3892/mmr.2016.5030

19. Xuan F, Jian J, Lin X, et al. 17-methoxyl-7-hydroxy-benzenefuranchalcone ameliorates myocardial ischemia/reperfusion injury in rat by inhibiting apoptosis and autophagy via the PI3K-Akt signal pathway. Cardiovasc Toxicol. 2017;17:79-87. doi:10.1007/s12012-016-9358-y

20. Sun J, Yu X, Huangpu H, Yao F. Ginsenoside Rb3 protects cardiomyocytes against hypoxia/reoxygenation injury via activating the antioxidation signaling pathway of PERK/Nrf2/HMOX1. Biomed Pharmacother. 2019;109:254-261. doi:10.1016/j.biopha.2018.09.002

21. Stellato M, Czepiel M, Distler O, Błyszczuk P, Kania G. Identification and isolation of cardiac fibroblasts from the adult mouse heart using two-color flow cytometry. Front Cardiovasc Med. 2019;6:105. doi:10.3389/fcvm.2019.00105

22. Chowdhury MA, Choi M, Ko W, et al. Standardized microwave extract of Sappan Lignum exerts anti-inflammatory effects through inhibition of NF- $\mathrm{kB}$ activation via regulation of heme oxygenase-1 expression. Mol Med Rep. 2019;19(3):1809-1816. doi:10.3892/ mmr.2019.9811

23. Wan YJ, Xu L, Song WT, et al. The ethanolic extract of caesalpinia sappan heartwood inhibits cerebral ischemia/reperfusion injury in a rat model through a multi-targeted pharmacological mechanism. Front Pharmacol. 2019;10:29. doi:10.3389/fphar. 2019.00029

24. Sorriento D, De Luca N, Trimarco B, Iaccarino G. The antioxidant therapy: new insights in the treatment of hypertension. Front Physiol. 2018;9:258. doi:10.3389/fphys.2018.00258

25. Moris D, Spartalis M, Tzatzaki E, et al. The role of reactive oxygen species in myocardial redox signaling and regulation. Ann Transl Med. 2017;5(16):324. doi:10.21037/atm

26. Cadenas S. ROS and redox signaling in myocardial ischemia-reperfusion injury and cardioprotection. Free Radic Biol Med. 2018;117:76-89. doi:10.1016/j.freeradbiomed.2018.01.024

27. Villeneuve NF, Lau A, Zhang DD. Regulation of the Nrf2-Keap1 antioxidant response by the ubiquitin proteasome system: an insight into cullin-ring ubiquitin ligases. Antioxid Redox Signal. 2010;13 (11):1699-1712. doi:10.1089/ars.2010.3211

28. Buendia I, Michalska P, Navarro E, Gameiro I, Egea J, León R. Nrf2ARE pathway: an emerging target against oxidative stress and neuroinflammation in neurodegenerative diseases. Pharmacol Ther. 2016;157:84-104. doi:10.1016/j.pharmthera.2015.11.003

29. Hybertson BM, Gao B, Bose SK, McCord JM. Oxidative stress in health and disease: the therapeutic potential of Nrf2 activation. Mol Aspects Med. 2011;32(4-6):234-246. doi:10.1016/j.mam.2011.10.006

30. Lee S, Choi SY, Choo YY, et al. Sappanone A exhibits anti-inflammatory effects via modulation of $\mathrm{Nrf2}$ and $\mathrm{NF}-\mathrm{\kappa B}$. Int Immunopharmacol. 2015;28(1):328-336. doi:10.1016/j.intimp.2015.06.015 


\section{Publish your work in this journal}

Drug Design, Development and Therapy is an international, peerreviewed open-access journal that spans the spectrum of drug design and development through to clinical applications. Clinical outcomes, patient safety, and programs for the development and effective, safe, and sustained use of medicines are a feature of the journal, which has also been accepted for indexing on PubMed Central. The manuscript management system is completely online and includes a very quick and fair peer-review system, which is all easy to use. Visit http://www. dovepress.com/testimonials.php to read real quotes from published authors.

Submit your manuscript here: https://www.dovepress.com/drug-design-development-and-therapy-journal 